\title{
PIBID: inserción de futuros profesores de matemáticas en el entorno escolar con vistas a minimizar el choque con la realidad ${ }^{1}$
}

DOI 10.26512/llc.v25i0.23110

\author{
Douglas da Silva Tinti \\ Universidade Federal de Ouro Preto - UFOP \\ Ana Lúcia Manrique \\ Pontifícia Universidade Católica de São Paulo - PUCSP
}

\section{Resumen}

El presente artículo tiene como objetivo analizar las acciones desarrolladas por un programa brasileño de iniciación a la docencia (PIBID), así como sus contribuciones a la minimización del futuro choque con la realidad vivido por los profesores en inicio de carrera como apuntan los estudios sobre esa etapa del desarrollo profesional. Para eso, fueron realizadas entrevistas y se pudo observar que la vivencia en el contexto escolar hizo la carrera docente más atractiva y contribuyó significativamente a la minimización del futuro choque con la realidad. En posesión de los datos colectados, concluimos que las políticas públicas orientadas a la formación de los profesores pueden contribuir para la superación del déficit de profesionales y propiciar espacios y experiencias formativas diferenciadas, que consideren la escuela como locus del aprendizaje docente.

Palabras clave: PIBID. Iniciación a la docencia. Inserción profesional. Formación de profesores.

1 Investigación financiada por la CAPES/Brasil. El presente trabajo es una ampliación de una comunicación dividida en las 17 Jornadas sobre el Aprendizaje y la Enseñanza de las Matemáticas (JAEM), Cartagena/España, 2015. 


\section{Resumo}

O presente artigo objetiva analisar as ações desenvolvidas por um programa brasileiro de iniciação à docência (PIBID) bem como suas contribuições para a minimização do futuro choque com a realidade vivenciado por professores em início de carreira como apontam os estudos sobre esta etapa do desenvolvimento profissional. Para tanto foram realizadas entrevistas e observou-se que a vivência no contexto escolar tornou a carreira docente mais atrativa e contribui significativamente para a minimização do futuro choque com a realidade. De posse dos dados coletados, concluímos que as políticas públicas voltadas à formação de professores podem contribuir para a superação do déficit de profissionais e propiciar espaços e experiências formativas diferenciadas, que considerem a escola como locus da aprendizagem docente.

Palavras-chave: PIBID. Iniciação à Docência. Inserção Profissional. Formação de Professores.

\section{Abstract}

The aim of this article is to analyze the actions developed by a Brazilian teaching initiation program (PIBID) as well as its contributions to the reduction of the future clash with the reality experienced by early career teachers, as the studies on this phase of professional development point out. Therefore, interviews were conducted and it was observed that the experience in the school context made the teaching career more attractive and contributed significantly to the decrease of the future clash with reality. Based on the collected data, we concluded that public policies aimed at teacher education can contribute to overcome the deficit of professionals and provide spaces and various formative experiences that consider the school as the place of teacher learning.

Keywords: PIBID. Initiation to Teaching. Professional Insertion. Teacher training. 


\section{Résumé}

Le présent article vise à analyser les actions développées par un programme brésilien d'initiation à l'enseignement (PIBID) ainsi que ses contributions à la minimisation du conflit futur avec la réalité vécue par les enseignants en début de carrière, comme le soulignent les études sur ce stade de développement professionnel. Pour cela, des entretiens ont été menés et il a été observé que l'expérience dans le contexte scolaire rendait la carrière d'enseignant plus attrayante et contribuait de manière significative à la minimisation du conflit futur avec la réalité. Sur la base des données collectées, nous concluons que les politiques publiques visant à la formation des enseignants peuvent contribuer à combler le déficit de professionnels et fournir différents espaces et expériences formatives qui considèrent l'école comme le lieu de l'apprentissage des enseignants.

Mots-clés: PIBID. Initiation à l'enseignement. Insertion professionnelle. Formation des enseignants.

\section{Introducción}

Desde 2007, en el escenario educativo brasileño, el Programa Institucional de Becas de Iniciación a la Docencia (PIBID) viene erigiéndose como una política pública dirigida a la formación de profesores. Dicho programa está adquiriendo cada vez más importancia no solo en el entorno escolar, sino también en el medio académico. En 2010 se aprobó un proyecto de la Pontificia Universidad Católica de São Paulo - PUC-SP, el cual se constituyó considerando cinco subproyectos segmentados por área de conocimiento. Uno de estos subproyectos es el PIBID Exactas PUC/SP, que comprende las carreras de Licenciatura ${ }^{1}$ en Matemáticas y Licenciatura en Física y desarrolla acciones formativas enfocadas a la Iniciación a la Docencia.

La Iniciación a la Docencia, entendida como la primera etapa del ciclo vital de los profesores (Huberman, 1992), corresponde a los dos o tres primeros años de enseñanza - y se caracteriza por los aspectos de supervivencia y descubrimiento, generalmente vividos paralelamente. La supervivencia se identifica como:

(...) el "choque de lo real", la constatación de la complejidad de la situación profesional: el tantear constante, la preocupación consigo mismo (“'Podré soportarlo?"), la distancia entre los ideales y la realidades del día a día del aula, la fragmentación del trabajo, la dificultad en hacer frente, simultáneamente, a la relación pedagógica y a la transmisión 
de conocimientos, la oscilación entre relaciones demasiado íntimas y demasiado distantes, las dificultades con los alumnos que crean problemas, con materiales didácticos inadecuados, etc. (Huberman, 1992, p.39).

Por su parte, el aspecto del descubrimiento es lo que, según Huberman (1992), permite que el profesor soporte el "choque con la realidad", pues «el entusiasmo inicial, la exaltación por estar, finalmente, en una situación de responsabilidad (tener su aula, sus alumnos, su programa), por sentirse en un determinado cuerpo profesional», son elementos que contribuyen a esta superación. (Huberman, 1992, p.39).

Con el objetivo de caracterizar el proceso vivido durante el(los) primer(os) año(s) de profesión, Silva (1997) señala que Müller-Fohbrodt y otros (1978) emplearon las expresiones reality shock ("choque con la realidad») y transition shock («choque de la transición»).

Para que los docentes que están en el inicio de sus carreras aprendan a gestionar los dilemas, propios de su actividad profesional, sin que estos se conviertan en una fuente de frustraciones, ansiedades o, en última instancia, en desmotivación profesional, es necesario que los profesores principiantes sientan la necesidad de elaborar y desarrollar -en consonancia con las características de la comunidad escolar en la que ejercen la profesión- su propio proyecto de formación continua que les permita, por medio de la transformación de su sistema de creencias, de la mejora de su autoconocimiento, de su autoestima y autoconcepto, abrirse más al cambio y desarrollarse personal y profesionalmente. (Silva, 1997, p.59).

Como podemos apreciar, la iniciación a la docencia es un periodo muy complejo. Todos los dilemas que viven los profesores durante este periodo apuntan a la necesidad de que pensemos en políticas públicas orientadas a ayudar a los profesores que están en el inicio de sus carreras para que se desarrollen profesionalmente.

Diversos países vienen desarrollando programas de iniciación con el objetivo de ayudar a los profesores principiantes en esta etapa profesional. Los programas de iniciación a la docencia pretenden ayudar al profesorado principiante en el ingreso a la profesión, buscando minimizar los efectos del "choque con la realidad":

Cuando alguien empieza la profesión de docente, teme la falta de adecuación de sus modos de pensar y actuar con los de sus colegas, no sabe a quién pedir ayuda ni cómo guiar sus procedimientos. Es como si, de la noche a la mañana, dejase repentinamente de ser estudiante y sobre sus hombros recayese una responsabilidad profesional, cada vez más creciente, para la que no parece estar preparado. (Silva, 1997, p.53).

Para ello, estos programas consideran un conjunto de factores que afectan directamente al trabajo que se realizará en el entorno escolar. En general, estos programas, que también pueden llamarse "programas de inducción», ofrecen apoyo y orientación, en la perspectiva de promover el aprendizaje y el desarrollo de la base 
de conocimiento profesional y ayudar en la socialización con la cultura escolar de los profesionales. (Ferreira y Reali, 2005).

Aunque se reconozca la importancia de estos proyectos para ayudar a la inserción del profesor novel, Ferreira y Reali señalaron que

En Brasil no hay una tradición de programas formativos de este tipo, lo que justifica que se lleven a cabo investigaciones sobre los motivos del escaso uso de dicha práctica, ni elementos que permitan comprender mejor esta clase de iniciativa y su inserción en el contexto brasileño. (Ferreira y Reali, 2005, P. 3)

Ante este hecho, podemos deducir que los profesores noveles se sienten desamparados, tanto por la universidad como por la propia comunidad escolar. Sus formadores tenían la preocupación de formarlos durante un período de tiempo (durante el grado). Al adentrar en una escuela, por lo general, no reciben el apoyo ni la orientación necesaria para superar los retos vividos durante el(los) primer(os) año(s) de actividad.

Este escenario parece que no solo predomina en Brasil. Silva (1997) señala que la misma situación se produce en Portugal:

En nuestro actual sistema de enseñanza, el profesor principiante se encuentra totalmente entregado a su suerte, sin una estructura de apoyo que le dé seguridad. Al contrario: se siente como si le evaluasen, le juzgasen, como si le mirasen con sospecha o indiferencia, lo que acabará reflejándose en la forma en cómo percibe su contacto con lo real. (Silva, 1997, p. 60).

En Brasil, el PIBID fue concebido para superar este escenario; no obstante, los sujetos en formación son profesores en formación inicial que viven un periodo que denominamos "predocencia» bajo la supervisión de un profesor de la universidad y de un profesor de la escuela pública.

En este contexto, proponemos los siguientes interrogantes: ¿qué acciones se desarrollan en el PIBID? ¿Estas acciones contribuyen a minimizar el futuro «choque con la realidad", vivido por los profesores que se encuentran en inicio de carrera? ¿De qué manera?

\section{Programa Institucional de Becas de Iniciación a la Docencia (PIBID)}

Venimos notando que, los últimos años, el Gobierno Federal brasileño viene intensificando la propuesta de políticas públicas dirigidas a la formación inicial de profesores. Ello puede estar ligado a la publicación del Decreto n. ${ }^{\circ} 6.755 / 2009$, que 
instituye la Política Nacional de Formação de Profissionais do Magistério da Educação Básica² (BRASIL, 2009).

De acuerdo con el referido decreto, esta política fue instituida con la finalidad de organizar - en régimen de colaboración entre el Gobierno Federal, los estados, Distrito Federal y los municipios de Brasil - la formación inicial y continua de los profesionales de magisterio para las redes públicas de educación.

Asimismo, en relación con la Política Nacional de Formación de Profesionales del Magisterio de Educación Básica, se decretó que la Coordinación de Perfeccionamiento de Personal de Nivel Superior (CAPES) incentivará, también, la formación de los profesionales de magisterio.

La CAPES incentivará la formación de profesionales de magisterio para que actúen en la educación básica, mediante el fomento de programas de iniciación a la docencia y concesión de becas a estudiantes matriculados en carreras de licenciatura en las instituciones de Educación Superior. (BRASIL, 2009).

Entre los programas incentivados por la CAPES, destacamos el PIBID, instituido en diciembre del 2007, por medio de una acción conjunta del Ministerio de Educación brasileño, por intermedio de la Secretaría de Educación Superior (SESu), de la CAPES y del Fondo Nacional de Desarrollo de la Educación (FNDE).

EI PIBID, cuya finalidad es apoyar la Iniciación a la Docencia de estudiantes de licenciatura, busca perfeccionar la formación de los docentes, valorizar el magisterio y contribuir al aumento del nivel de calidad de la Educación Básica. Este documento señala los siguientes objetivos de este Proyecto de Iniciación a la Docencia:

I. incentivar la Formación de Profesores para la Educación Básica, contribuyendo a aumentar la calidad de la escuela pública;

II. valorizar el magisterio, incentivando a los estudiantes que optan por la carrera docente;

III. aumentar la calidad de las acciones académicas dirigidas a la formación inicial de profesores en las carreras de licenciatura de las instituciones públicas de educación superior;

IV. introducir a los estudiantes de licenciatura en el día a día de las escuelas de la red pública de educación, promoviendo la integración entre Educación Superior y Educación Básica;

V. proporcionar a los futuros profesores la participación en experiencias metodológicas, tecnológicas y prácticas docentes de carácter innovador e interdisciplinar y que busquen la superación de los problemas identificados en 
el proceso de enseñanza-aprendizaje, teniendo en cuenta el desempeño de la escuela en evaluaciones nacionales, tales como Provinha Brasil, Prova Brasil, SAEB y ENEM, entre otras;

VI. incentivar a las escuelas públicas de Educación Básica, convirtiéndolas en protagonistas en los procesos formativos de los estudiantes de las diferentes licenciaturas, movilizando a sus profesores como coformadores de los futuros profesores. (CAPES, 2010)

Con base en el Informe de Gestión (2009-2013) presentado por la CAPES, fueron ofrecidas, en ese período, 26.918 becas. La segmentación de esas becas se presenta en la Tabla siguiente:

Tabla 1: Total de becas distribuidas por el PIBID en el período de 2009 a 2013 segmentada por modalidad.

\begin{tabular}{|l|l|l|l|}
\hline Modalidad & Total de becas & $\%$ \\
\hline Becario de Iniciación a la Docencia & 72.845 & 80,7 \\
\hline Supervisor & 11.717 & 13,0 \\
\hline Coordinador de Área & 4.924 & 5,5 \\
\hline Coordinador Institucional & 455 & 0,5 \\
\hline Coordinador de Gestión & 313 & 0,3 \\
\hline Total & 90.254 & 100,0 \\
\hline
\end{tabular}

Fuente: Elaborado por los autores con base en el Informe de Gestión (2009-2013) (CAPES, 2013).

Es importante señalar también que, según el Informe de Gestión (2009-2013) presentado por la CAPES, a lo largo de este período, se ha invertido casi 747 millones de reales en el desarrollo de los proyectos propuestos por las Instituciones de Educación Superior (IES) en el ámbito del PIBID.

Inicialmente, el PIBID se destinaba a los alumnos de licenciatura de instituciones federales. De acuerdo con el Edicto CAPES N 018/2010, la propuesta se extendió a las universidades y centros universitarios comunitarios, confesionales y filantrópicos.

Ante esta extensión, en 2010, la PUC/SP propuso un Proyecto Institucional de Becas de Iniciación a la Docencia titulado «Procesos de formación inicial de profesores en contextos colaborativos: docencia y prácticas educativas desarrolladas en escuelas públicas del estado de Sao Paulo», y esta propuesta fue aprobada. 


\section{PIBID - PUC/SP}

El PIBID - PUC/SP se compone de cinco subproyectos, entre los cuales destacaremos a continuación el subproyecto PIBID Exactas - PUC/SP, compuesto por las carreras de Licenciatura en Matemáticas y Licenciatura en Física.

Para la implantación del PIBID Exactas - PUC/SP se consideraron dos escuelas colaboradoras. En cada una de estas escuelas se eligió a un profesor-supervisor para seguir los trabajos de los becados (alumnos de las carreras de Matemáticas o Física de la PUC/SP) durante un periodo de 20 horas semanales de trabajo. Para este seguimiento, el profesor-supervisor percibe una dotación económica mensual denominada «beca del supervisor».

Para el desarrollo del proyecto se seleccionaron diez becados de Licenciatura en Matemáticas y diez becados de Licenciatura en Física. Estos becados dedican 20 horas semanales de trabajo y perciben una dotación económica mensual denominada «beca del estudiante de licenciatura».

Es importante destacar que, desde su implantación, no se ha producido ningún cambio en el efectivo de profesores-supervisores. Sin embargo, el efectivo de becados sí sufrió modificaciones por diversos factores, como, por ejemplo, la conclusión de la carrera o el incumplimiento de las actividades previstas en el subproyecto PIBID.

Las actividades desarrolladas en el PIBID Exactas - PUC/SP provinieron de las metas establecidas cuando se propuso el proyecto a la CAPES, y se desarrollaron entre agosto de 2010 y julio de 2013.

Las actividades desarrolladas se derivaron de las metas establecidas en el momento de la proposición del proyecto al edicto de la CAPES/INEP, y fueron desarrolladas en el período de agosto de 2010 a julio de 2013. Para eso, se establecieron tres metas, a saber: conocimiento de la escuela, proyectos de intervención y evaluación del proyecto. Más allá de eso, se desarrollaron acciones de divulgación de los resultados a las comunidades científica y escolar, además de la elaboración de informes de rendición de cuentas a la CAPES.

A continuación describimos una de las metas que se establecieron, buscando presentar las contribuciones que identificamos para la formación inicial de los becados.

- Conocimiento de la escuela: en esta primera etapa se buscó describir y analizar la realidad escolar con el objetivo de ampliar el conocimiento de los futuros profesores acerca de la comunidad escolar.

Para alcanzar este objetivo se propuso la realización de observaciones y análisis no solo de las características socio-político-económicas y culturales de los alumnos, del cuerpo docente y de la zona de la escuela, sino también de las características de la comunidad escolar. Esta actividad ocurrió durante los meses entre octubre y diciembre de 2010. 
Con estos datos, los becados elaboraron un informe analítico exhaustivo, presentando características locales y reflexionando, también, sobre el desempeño de los alumnos de las escuelas en evaluaciones externas. Esto proporcionó una serie de informaciones para proponer las acciones que se realizaron a lo largo del subproyecto PIBID Exactas - PUC/SP.

Por considerar la inserción de los becarios de iniciación a la docencia en el futuro ambiente de trabajo, el PIBID Exactas - PUC/SP les proporcionó una percepción más amplia del contexto escolar. Después de conocer las escuelas $A$ y $B$, los futuros profesores percibieron las distintas condiciones de trabajo que un profesor puede encontrar, y además de eso, reflexionaran sobre el papel de la gestión escolar en el desarrollo del trabajo docente.

En esa vivencia, los futuros profesores pudieron identificar diferencias entre escuelas públicas estatales y municipales, además de reflexionaren sobre los impactos de esas diferencias en el cotidiano escolar. Esta identificación es un factor que contribuye a la formación, puesto que pueden ingresar en la carrera docente en una de estas realidades.

Esa acción de conocer el contexto escolar, identificar los niveles de gestión, conocer el contexto histórico, el público objetivo, la comunidad alrededor de la escuela y su estructura física, se hizo necesaria por el hecho del PIBID Exactas - PUC/SP considerar la escuela como un espacio formativo en el proceso de Aprendizaje de la Docencia de sus futuros profesores.

En esta inmersión en el contexto escolar, también se puede destacar los descubrimientos y las experiencias que los futuros profesores tuvieron con relación a los procedimientos burocráticos de la escuela, a los documentos que la orientan, sus espacios colectivos de formación y a su público objetivo.

Es perceptible que esa vivencia en el PIBID Exactas - PUC/SP, de conocer la realidad escolar, fue un momento que ha dejado huellas en la formación de los futuros profesores, y que esa acción los impulsa a optar por esa carrera y no desanimarse ante los dilemas y desafíos que podrán enfrentar en los primeros años de la carrera docente.

De ese modo, podemos percibir que el principal objetivo del PIBID Exactas - PUC/SP en esta primera etapa ha sido alcanzado. Consideramos que esta puede ser apuntada como una de las grandes contribuciones de esa primera meta.

En ese sentido, es posible inferir que conocer el contexto escolar en un período de "pre-docencia" puede ser un factor que contribuya para la minimización del "choque con la realidad", con miras a la identificación, reflexión y vivencia de algunos de los desafíos que un profesor puede enfrentar en su cotidiano.

También es importante señalar que, con el acúmulo de esa vivencia en el contexto 
escolar, los futuros profesores produjeron un informe presentando, así, los descubrimientos que hicieran a lo largo de esa primera etapa del PIBID Exactas PUC/SP. Este informe presenta aspectos: físicos, pedagógicos, humanos, históricos, además de la intersección entre eses aspectos.

Los futuros profesores comprenden esa acción como una contribución a la escuela, puesto que este documento ha sido producido a partir de ella, con ella y para ella. Este informe también ayudará los nuevos becarios del PIBID PUC/SP que fueren insertados en etapas posteriores a del conocimiento del contexto escolar.

Percibimos que esa vivencia fue significativa a los futuros profesores, puesto que las puertas de la escuela estaban abiertas a que ellos pudiesen entrar y sentirse parte integrante. Lo mismo debería suceder en los cursos de formación inicial, cuando los futuros profesores empiezan la Práctica Supervisada, pero sabemos que, en muchos casos, la realidad es muy distinta de la que relatamos hasta aquí.

Delante de lo expuesto, se queda evidente que conocer la realidad escolar hizo con que los involucrados confirmasen el deseo de formarse profesores y de ejercer la docencia como profesión. Esa vivencia en el PIBID Exactas - PUC/SP ayudó a los futuros profesores a superar posibles impresiones y vivencias negativas que tuvieron en la escuela pública, hecho que puso en cuestionamiento las ideas de "ser profesor" y de "ser profesor en escuela pública".

En ese contexto, resaltamos la importancia de las Alianzas Oficiales (Foerste, 2005) en el desarrollo de acciones formativas que contribuyen para el aumento del atractivo, además de posibilitaren una vivencia de formación distinta - considerando la escuela como locus de la Aprendizaje de la Docencia.

Es importante señalar que esta meta se constituyó en objeto de investigación de la disertación de maestría desarrollada por Tinti (2012). Él concluyó que, además de vivenciar los dilemas del cotidiano escolar, esta meta desencadenó reflexiones sobre el futuro y posible ambiente de trabajo, contribuyendo, así, para la superación de posibles preconceptos acerca del sistema público de enseñanza brasileño. También apuntó que esa vivencia en el PIBID Exactas - PUC/SP puede hacer la carrera docente más atractiva a los alumnos que cursaron licenciatura, además de contribuir a la minimización del "choque con la realidad" (Silva, 1997) en los primeros años de profesión, puesto que, en ese período, los futuros profesores vivieron muchos de los dilemas emergentes del contexto escolar.

Con todo, apreciamos que esta vivencia fue significativa para los futuros profesores, puesto que tenían las puertas de la escuela abiertas para poder entrar y sentirse parte de ella. 


\section{Recorrido metodológico}

El objetivo del presente artículo es analizar e indicar las contribuciones de las acciones desarrolladas por el PIBID Exactas PUC/SP para minimizar el futuro choque con la realidad que viven los profesores durante el inicio de sus carreras.

Para ello, se optó por analizar la primera etapa del proyecto, titulada: conocimiento de la escuela. Para alcanzar el objetivo propuesto consideramos, como instrumento de recogida de datos, la entrevista semiestructurada. El guion para su realización se elaboró después de analizar las propuestas iniciales del PIBID Exactas - PUC/SP.

Para seleccionar los sujetos de estudio, utilizamos el siguiente criterio: identificar, entre los becados del PIBID Exactas - PUC/SP, a los alumnos de Licenciatura en Matemáticas que estuvieran en el programa desde su implantación. Así, seleccionamos a tres sujetos a los que denominaremos Júlia, Lucas y Diogo. En el siguiente cuadro presentamos el perfil de los sujetos de estudio.

Cuadro 1: Características de los sujetos de estudio.

\begin{tabular}{|l|l|}
\hline Sujeto & Perfil \\
\hline Júlia & $\begin{array}{l}\text { Tiene 42 años, hizo toda su Educación Básica en una escuela pública. } \\
\text { Siempre quiso ser profesora de matemáticas. Por eso, } 21 \text { años después } \\
\text { de terminar Educación Secundaria, se matriculó en la Licenciatura en } \\
\text { Matemáticas. }\end{array}$ \\
\hline Lucas & $\begin{array}{l}\text { Tiene } 49 \text { años, hizo toda su Educación Básica en una escuela pública. Se } \\
\text { licenció en Ciencias y estudió tres años de Ingeniería antes de empezar la } \\
\text { Licenciatura en Matemáticas. }\end{array}$ \\
\hline Diogo & $\begin{array}{l}\text { Tiene 32 años, hizo toda su Educación Básica en una escuela pública. } \\
\text { Estudió Enseñanza Secundaria en la modalidad «EJA» (Educación de } \\
\text { Jóvenes y Adultos). }\end{array}$ \\
\hline
\end{tabular}

Fuente: Entrevistas realizadas.

Aunque la entrevista pueda emplearse para diferentes fines, nos atendremos aquí a describirla como instrumento de recogida de datos para la producción de conocimiento científico, en una perspectiva cualitativa de investigación. Entendemos que las buenas entrevistas se caracterizan por el hecho de que los sujetos se encuentren a gusto y expongan libremente sus puntos de vista, y producen una riqueza en los datos, llenos de palabras que revelan las perspectivas de los entrevistados (Bogdan y Biklen, 1994).

Además de conocer un poco más sobre la trayectoria formativa y personal de Júlia, 
Diogo y Lucas, las entrevistas nos posibilitaron detectar las percepciones que tuvieron sobre las acciones iniciales desarrolladas en el PIBID Exatas - PUC/SP y, de esta forma, fue posible investigar las contribuciones de esta acción al proceso formativo de los sujetos y, así, responder a los interrogantes planteados acerca de la minimización del choque con la realidad.

Las entrevistas se realizaron individualmente a principios del primer semestre de 2011 , en días diferentes. Cada entrevista duró un promedio de 35 minutos. Júlia fue la primera entrevistada, seguida de Diogo y de Lucas. Se grabaron y transcribieron todas las entrevistas, con el permiso de los sujetos, que sirvieron de apoyo para el análisis que presentamos a continuación.

\section{Análisis de datos}

Aunque el PIBID Exactas - PUC/SP no sea una acción de Prácticas Supervisadas, la naturaleza y las características de las actividades desarrolladas por los futuros profesores se acercan mucho a una conducción deseada para la realización de Prácticas Supervisadas, puesto que propone la superación de la Colaboración Dirigida (Foerste, 2002) en donde la universidad es quien determina las acciones que se desarrollarán y la escuela es quien, meramente, ejecutará dichas acciones.

Este tipo de relación puede acarrear pérdidas para los futuros profesores, ya que la escuela no está invitada a pensar, colaborativamente, la formación de estos profesores. De este modo, los futuros profesores solo pueden conocer una parte de la escuela: el aula. En otros casos, las puertas de la escuela ni siquiera estarán abiertas, como podemos observar en las palabras de Lucas:

«Así que creo que la gran ventaja del PIBID es eso, que te da otra visión. Porque, normalmente, cuando eres alumno universitario, vas a hacer unas prácticas, y es difícil, no lo consigues; yo, al menos, no lo conseguí. Fui a varias escuelas y no conseguía hacer las prácticas. Entonces fui a una escuela pública que no estaba mal, una pública con unos principios bien fundamentados. Llegué y Coordinación me dijo: “¿dónde te tengo que firmar las prácticas?" A lo que le dije: "no, no quiero que simplemente me firme el contrato de prácticas, lo que quiero es entrar en el aula. Quiero ver la realidad".» (Lucas, entrevista realizada el 21/03/2012)

Al entender que estos futuros profesores pasarán por el periodo de Iniciación a la Docencia (Tancredi, 2009; Gama, 2007), y que durante este periodo podrán sufrir un "choque con la realidad» (Huberman, 1992; Silva, 1997), señalamos la necesidad de que los cursos de formación inicial de profesores piensen estrategias para minimizar este impacto con el contexto escolar. 
Como hemos dicho anteriormente, este choque puede atenuarse cuando posibilitamos a los futuros profesores el conocimiento del contexto escolar, los procedimientos que sigue la escuela y la forma en cómo los profesores con más experiencia dirigen sus clases, pero ese conocimiento no debe restringirse a una teoría o a una práctica, sino que debe estar previsto en la articulación teórica y práctica.

"[...] yo ya conocía la realidad de las escuelas públicas, me parece que algunos alumnos de aquí del PIBID no la conocían y sufrieron un choque, porque se pensaban que otra cosa» (Diogo, entrevista realizada el 19/03/2012)

Aunque se reconozca que estos futuros profesores ya pasaron un tiempo considerable en la escuela - como alumnos de la Educación Básica - y que, habida cuenta de ello, ya la conocen, hay que resaltar que la visión que tienen sobre la escuela es una visión de alumno (y, por lo tanto, limitada). De este modo, pensar en acciones que propicien esa toma de consciencia del contexto escolar posibilita una ampliación de esta visión y puede, también, posibilitar la mejora de la calidad de la enseñanza, puesto que las acciones se planifican considerando las fortalezas y las debilidades de los alumnos que están en las escuelas de Educación Básica.

Como hemos señalado anteriormente, esta acción se desarrolló en el PIBID Exactas - PUC/SP y nos percatamos de que fue de gran utilidad para los futuros profesores, principalmente por el hecho de tener que desarrollar, en un momento posterior, proyectos de intervención:

«[...] el objetivo era precisamente que uno tuviera el primer contacto, el primer contacto con la escuela, porque nadie iba a llegar hablando de un plan de intervención desconociendo al alumnado, pues conocer al alumnado es importante. El estudio del alumnado lo hizo otra pareja, pero nos sirvió mucho para el plan de acción y de intervención porque, a partir del momento en el que conoces al público con el que trabajas, desarrollas un plan adecuado para ese tipo de personas, de público." (Diogo, entrevista realizada el 19/03/2012)

En esta vivencia, los futuros profesores pudieron superar una visión prejuiciosa de las escuelas públicas y, además, reflexionar sobre su elección profesional:

«Nos preguntábamos, ¿esto está mereciendo la pena? A parte de la beca, del valor económico de la beca, ¿qué otro valor estamos nos estamos llevando a cambio? [...] Así que, para mí, la principal contribución del PIBID, si me fuera mañana, será esta: ¿vale la pena dedicarle tiempo a los alumnos? ¿Sí o no? Si sí, eres un profesor; si no, déjalo y búscate otra cosa». (Diogo, entrevista realizada el 19/03/2012)

Podemos notar que este tipo de reflexión, de cierta manera, puede ser decisiva para que el futuro profesor elija o no esta profesión. Entendemos que las acciones iniciales del PIBID Exactas - PUC/SP fueron importantes para los futuros profesores, sobre 
todo por la relación que lograron establecer entre la vivencia en el PIBID Exactas PUC/SP y las discusiones teóricas realizadas en el curso de formación.

«Sí, estoy aprendiendo mucho con relación a la práctica. Como te he dicho antes, allí solo nos centramos en la práctica. Por eso, los trabajos que solemos hacer en la facultad, relacionados con las asignaturas pedagógicas, los hago con mucha facilidad, porque es lo que venimos haciendo desde el principio, ya estoy familiarizada, así que no me suponen ninguna dificultad las asignaturas pedagógicas, debido a la vivencia en el PIBID, creo. [...] He aprendido mucho en la práctica, probando. Tuve acceso al "Proyecto PolíticoPedagógico" de la escuela, lo leímos e hicimos un estudio; por algo saqué un diez en esa asignatura, porque ya sabía todo». (Júlia, entrevista realizada el 21/03/2012)

Esto también se va haciendo evidente a medida que nos damos cuenta de la influencia de esta etapa en las acciones que los futuros profesores desarrollan en otros espacios formativos:

«[...] hice las prácticas en Secundaria. Todo lo que hicimos en el PIBID lo apliqué en las prácticas y supuso la contribución a la escuela que el director quería. Como el director me abrió las puertas, él no quería que simplemente me quedase mirando; quería que hiciese un proyecto, así que hice una evaluación diagnóstica, la apliqué y después cogí las dificultades de los alumnos y a partir de ahí y las trabajé, di algunas clases de refuerzo. Así pues, todo lo que hice en el PIBID lo pude aplicar en las prácticas, así que veo que todo lo que vengo aprendiendo en el PIBID es la praxis de un profesor en su día a día, a diferencia de la carrera, se complementan, la teoría y la práctica, y el PIBID, en general, es la práctica de todo aquello que vengo aprendiendo en la carrera.» (Júlia, entrevista realizada el 21/03/2012)

Aparte de esta relación teórica y práctica, los futuros profesores señalan, que acciones como las desarrolladas en el PIBID Exactas - PUC/SP, pueden contribuir a mejorar la enseñanza en la Educación Básica e incluso en los cursos de formación de profesores:

«[...] creo que la tendencia es que mejore, principalmente por eso, debido a esos proyectos. Vemos que, antes, nadie se preocupaba. La propia CAPES no se preocupaba en invertir dinero en la formación de alumnos de licenciatura en final de carrera para que conozcan el lugar donde se supone que trabajarán, antes de que entren.» (Diogo, entrevista realizada el 19/03/2012)

Este testimonio nos remite a la Política Nacional de Formação de Profissionais do Magistério da Educação Básica (Brasil, 2009), instituida por el Decreto 6.755, en el que queda claro el papel de la CAPES en relación con la Formación de Profesores:

Art. 10. La CAPES incentivará la formación de profesionales de magisterio para trabajar en la Educación Básica, mediante el fomento de programas de iniciación a la docencia y concesión de becas a estudiantes matriculados en cursos de licenciatura en las instituciones de Educación Superior. (Brasil, 2009). 
Es evidente que un proyecto desarrollado por medio de un convenio UniversidadEscuela posibilita una vivencia significativa y pueda potenciar la formación inicial de los profesores, y puede ser un factor que contribuya a minimizar el posible choque con la realidad durante los primeros años de docencia.

EI PIBID hizo posible que se superaran algunas ideas preconcebidas negativas que sufre la escuela pública y que hacen que la carrera profesional en esta institución sea poco atractiva. En este contexto, salta a la vista la influencia de las políticas públicas dirigidas a la formación inicial con el objetivo de contribuir a que las personas se sientan más seducidas por la carrera docente.

\section{Consideraciones finales}

Al reflexionar sobre las aportaciones del PIBID a los futuros profesores de matemáticas, teniendo como base la primera etapa de implantación del PIBID Exactas - PUC/SP, podemos destacar que este programa propició un acercamiento entre la teoría vivida en la licenciatura y la práctica vivida en el contexto escolar, contribuyendo, así, a superar la dicotomía teoría-práctica y a enfrentar el futuro choque con la realidad profesional.

Inseridos en el contexto escolar, los futuros profesores percibieron la dinámica, los procedimientos burocráticos ejecutados por los profesores de las escuelas públicas y el proceso de gestión escolar. Los futuros profesores percibieron, asimismo, la importancia de que el profesor conozca bien a su alumnado para poder planificar sus clases.

La «vivencia de la práctica» fue un gran punto fuerte del PIBID Exactas de la PUC/SP. En este subproyecto, los alumnos pudieron demostrar efectivamente esta característica al preparar y organizar actos de difusión de las ciencias y situaciones didácticas implantadas en clase, que hicieron posible un contacto mayor y más directo con las dificultades de aprendizaje de sus alumnos, así como un enfoque diferente para ellos. Estos momentos facilitaron a los alumnos becados el conocimiento del público escolar y de las situaciones del día a día de este entorno. Además, sobre la experiencia de participar en el PIBID Exactas PUC/SP, conforme afirma Manrique:

Asimismo, se puede decir que esa experiencia contribuyó a confirmar la elección profesional y posibilitó el acercamiento entre la teoría estudiada en la licenciatura y la práctica vivida en el contexto escolar. Y, con más firmeza, que el trabajo colectivo e interdisciplinar no es una tarea fácil. Y, por último, este proyecto de intervención les propició a los alumnos de las carreras de licenciatura la oportunidad de considerar a la escuela como «lugar» del aprendizaje de la docencia y pensar sobre la función de enseñar. (Manrique, Manrique, 2012, p.70) 
Para los becados, el PIBID se mostró eficaz en cuanto a la aplicación de propuestas experimentales y organizativas, ya que fueron acciones sincronizadas con la propuesta pedagógica del centro de enseñanza. Además, las acciones desarrolladas contaron con la colaboración de todos los implicados: universidad, becados y escuela.

En este contexto, destacamos como un factor que contribuyó a la formación de los futuros profesores la superación de la visión prejuiciosa que tenían del sistema público de enseñanza, hecho que contribuía, también, al desprestigio de la carrera profesional en este tipo de enseñanza.

Finalmente, entendemos que la principal contribución que el PIBID Exactas - PUC/ SP proporcionó a los futuros profesores fue la de concebir un espacio formativo diferenciado, que considera a la escuela como «lugar» del aprendizaje de la docencia. Por medio de este convenio oficial, los futuros profesores pudieron vivir un periodo que entendemos como pre-docencia, en el que fue posible proyectarse en una futura profesión docente, viviendo sus dilemas y desafíos. Con todo, esperamos que, a corto plazo, las acciones desarrolladas por los diferentes proyectos PIBID desarrollados en Brasil contribuyan a minimizar el déficit de profesores y a mejorar la calidad de la educación y de los cursos de formación de profesores en el territorio nacional.

\section{Referencias bibliográficas}

Bogdan, R. C.; BIKLEN, Sari Knopp. (1994) Investigação Qualitativa em Educação. Porto: Porto Editora.

Brasil. Decreto No 6.755 - Política Nacional de Formación de Profesionales del Magisterio y de la Educación Básica - de 29 de enero de 2009. Disponible en: http://www.planalto.gov.br/ccivil_03/_ato2007-2010/2009/Decreto/D6755. htm>. Consulta: 2/01/ 2017.

Capes. Edicto No 018/2010/CAPES. Disponible en: http://www.capes.gov.br/ educacao-basica/capespibid>. Consulta: 21/2/2017.

Capes, Brasil. PIBID, Informe de Gestión. Extraído el 3/2016, de la Dirección de Formación de Profesores de la Educación Básica DEB: 2013. http://capes.gov.br/ images/stories/download/bolsas/1892014-relatorio-PIBID.pdf

Ferreira, L.A.; Reali, A.M. de M. R. Aprendendo a ensinar e a ser professor: contribuições e desafios de um programa de Iniciação à Docência para professores de educação física. Anais de la $28^{\text {a }}$ Reunión anual de ANPEd. Caxambu: ANPEd, 2015. 
Foerste, E. Parceria na formação de professores. São Paulo: Cortez, 2005.

Gama, R. P. Desenvolvimento Profissional com apoio de Grupos Colaborativos: o caso de professores de Matemática em início de carreira. Tesis (Doctorado en Educación Matemática), FE-Unicamp, Campinas, 2007.

Huberman, M. (1995) O ciclo de vida profissional dos professores. In: Nóvoa, A. (org.). Vidas de Professores. Porto: Porto Editora.

Manrique, A.L. (2012) Iniciação à Docência: uma experiência de estágio em licenciatura de matemática e física. Coleção Textos FCC, n. 35, p. 63-127.

Silva, M. C. M. (1997) O primeiro ano de docência: o choque com a realidade. In: ESTRELA, M.T. (org.). Viver e construir a profissão docente. Porto: Porto Editora, p. 51-80.

Tancredi, R. P. (2009) Aprendizagem da docência e profissionalização: elementos de uma reflexão. São Carlos: EdUFSCar.

Tinti, D. da S. (2012) PIBID: um estudo sobre suas contribuições para o processo formativo de alunos de Licenciatura em Matemática da PUC-SP. Tesina (Máster en Educación Matemática) - Programa de Estudios Posgraduados en Educación Matemática, Pontifícia Universidade Católica de São Paulo, São Paulo. 